\title{
Analysis of Computer Software Development and Database Management
}

\author{
Shan Jiang ${ }^{1, \text { a }}$ \\ ${ }^{1}$ Information Technology Institute, Henan University of Chinese Medicine, Zhengzhou, Henan, \\ China, 450000 \\ aemail,
}

Keywords: Analysis, Computer Software Development, Database Management

\begin{abstract}
Computer software development has been used in many areas and it plays an important role in improving the efficiency. Computer software design is generally divided into four steps: analysis, design, testing and maintenance, which must meet the needs of customers. This paper briefly describes the problems in computer software development and database management and discussed the measures to solve the problem, hoping provides some reasonable recommendations to solve the issues of computer software development and database management in current stage.
\end{abstract}

\section{Introduction}

Modern society has a certain dependence on the computer. Whether it is game entertainment, or file transfer, information sharing have to rely on the computer to complete, almost every day in the use of modern computing, the use of computers to obtain information, computers enrich the lives of mankind, the computer in modern society more and more important. Computer is a great success, no doubt brought by computer software, there is no computer software, the role of the computer is out of the question and the computer software is the soul of the computer. On the other hand, the database management, not only for the database to run to create favorable conditions to ensure the smooth operation of the database, but also improve the dissemination of information and improve the quality of information management. If the computer software is the heart of the computer, then the database management is the computer's blood, the two are to protect the computer use, improve computer performance key.

\section{The Concept and Basic Processes of Computer Software Development}

Computer software development has been used in many areas in improving work efficiency plays an important role. In general, the computer software development can be understood from two aspects, namely the computer system software and computer application software. Among them, the former is to solve some practical problems and development work, mostly for all types of software updates and functional innovation. Through the development of computer software system software, can strengthen the database management system, operating system management and maintenance. The latter is compared to the computer system software development, technology upgrade level is more intuitive, more operational, better reflect its function and value. In today's society, the development of computer software not only reflects the general needs of people, more and more reflect the needs of the public, to achieve the perfect combination between the two.

The basic flow of computer software development consists of three parts, namely, software design, programming and system testing. Among them, the first part of the software design process, is the most basic part of the whole process, directly determines the software development level and application level. Software design links usually include the functional design, the overall structure design, module design, in addition, including programming, debugging and submission procedures and so on. The second link is the process of writing links. This link is mainly based on software design to complete the preparation of the program. This link in the computer software development process is very important, accounting for the entire process of software development about $30 \%$ of the time. Smooth coding work is the key to improve the efficiency of software development work. The third link is the software testing link. After the software writing process is completed, the 
software is tested to ensure its function should be achieved to ensure the stability of an important part of its operation.

The value of computer software development is embodied in its ability to meet the initial needs of software design. Therefore, for the computer software development, the needs analysis is the primary consideration. No demand for software systems, even if the development is also no use of value, and a waste of time and energy software developers. The purpose of computer software development is to ensure that its functions can be achieved to ensure that it can meet certain needs, and have a certain degree of reliability, convenience and ease of use. Then, the real value of computer software development is to meet the public demand for the majority of users, and in software development fully embodies the universality and popularity. The user-recognized software is the practical, valuable software. It is also important to ensure that the software development process is as professional and pipelined as possible to ensure that it has sufficient software infrastructure, hardware infrastructure, and technical support to assist the developer in software development, to maximize the value of software development, to the maximum extent possible.

\section{The Contents and Its Role of Database Management}

Set the present situation as the main basis, mainly include the following: First, the existence of its own procedures and related data have a certain degree of independence, the data presented in the process of data model will be used in the way there will be related to that Out, while containing the relevant characteristics of the data. Second, the basic data management in the process of a department or business as an independent entity, and as a basis for the establishment of a comprehensive and systematic database system, the data used for the management and control. Third, the existence of its own user interface is an ordinary type, the relevant user data development and utilization, will obtain certain advantages. Fourth, the actual situation as a specific reference standard management related applications, and through the database to find relevant and have practical information, to further reduce the storage process of duplication of storage occurs; In addition, the establishment of the new data management Structure can be effective, comprehensive use of data.

Improve the Data Utilization. Through the development of the database and the characteristics of the analysis can know that in fact, the database is an intelligent data "warehouse", people can store and control data through the database to improve data processing efficiency. But a huge amount of information must have the appropriate management and control, so database management is also very important. A database is not just about storing data. In today's huge amount of data progress, if you do not do a good job of database management, database functions and functions will be difficult to effectively play, some of the necessary access is difficult to achieve. Database management is to optimize the data storage, data redundancy, to avoid duplication of data an important means and the main way. Do a good job of database management, even if the amount of data, but also to cope with ease, the data utilization will be greatly improved.

Data Integration. The purpose of the database is to improve data utilization, data security, reliable, fast processing and storage. If the data cannot be effectively used, then the database storage will lose its meaning. Database management not only protects the data value, but also enhances the data management level through the integration of data resources. The integration of data resources of database system is more efficient and intelligent. On the other hand, data management is more data sharing to create the conditions for data sharing is to improve data utilization, so that the value of data is further sublimation. Under the database management, the database can provide data to all the people who have the authority to realize the multi-user extraction, query, call, use, so that the data more flexible, so that the entire database to meet the needs of more users.

Ensure the Data Quality Safety. Database management prevents damage and loss of data. Database management not only provides security in data security, but also can judge the completeness of data through data fault diagnosis, and take some recovery behavior, not only guarantee the data integrity, but also ensure the data quality and reliability. On the other hand, through database management, you can achieve the data in the database encryption, encryption is to 
protect data security and an important means of textile leakage, the enterprise data security is essential. The purpose of database management is not only to improve data utilization, to ensure data integrity, more importantly, to protect the security of data. Many modern data contains a large number of trade secrets, in the event of leakage is bound to cause huge economic losses related businesses, database management is significant.

\section{The Currently Exists Related Issues of Database}

There is a human problem in the process of managing the database. For example, the relevant management personnel in the database during the operation of the method used inappropriate, not in accordance with the relevant safety management standards, resulting in the process of managing the database of different types of security problems.

There are problems in carrying out the work of the database system, which led to the emergence of a series of problems in the process. For example, with the current development and innovation of network technology, its own security has been greatly challenged and the management process will inevitably be the corresponding problems.

There are problems in the operation of the database system. The results of the current practice show that the problem is the main reason for the emergence of information disclosure database. At the same time, with the operation of the user there is a certain non-normative, so that the computer by the invasion of the virus. With the outbreak of these viruses, the data and information will be exposed, so that the calculation of the operation and work are seriously affected. In addition, computer users in the daily operation, some hackers will take the opportunity to attack the database, modify and destroy the contents of which, so that data leakage.

\section{The Approaches Taken in the Database Management}

Security Policy. It is necessary to continuously improve the safety of the part of the data, as far as possible to prevent the phenomenon of data modification and illegal access. After the implementation of the measure, although the system is subject to a certain degree of damage, but stored in the relevant data is spread out and the possibility of theft is not very large. Throughout the security system, the need to establish a comprehensive authentication mechanism to ensure that users and server data docking, be prepared to verify the identity information and other data, in order to avoid illegal or other inappropriate means of intrusion background procedures, and limit and suppress interference Destroy the behavior of the entire system, so that the other party cannot enter the system core, undermining system security. The specific implementation method can be used by WPKI or Keberos triple encryption authentication and other methods for user security management. As a result, users can be a good identity to identify and identify, strengthen security.

Manage Policies. In the use of the database process, we must always maintain awareness of prevention, increase their vigilance, and fundamentally eliminate information leakage incidents. Using the methods mentioned before, you can make the database security is enhanced, but also to ensure that the legitimate rights and interests of users. The most effective way to enhance data security is to maintain it as necessary. In the process of database maintenance and management, you must back up the necessary information, so even if there are hackers, it is difficult to have an opportunity. Although the database through a number of security tools to a certain extent, improve the security level, but still cannot put an end to security incidents. Therefore, it is necessary to strengthen the management mechanism, which can be managed by virtual server and dynamic distributed database management. Only in this way can the normal operation of the database be ensured and the data protection can be timely taken when the accident happens.

Maintenance Policy. At present, database maintenance has also become one of the important objects to be considered in the development of computer software. In addition to a variety of protection means, a safe system needs to have excellent system maintenance and management. The database maintenance level depends on whether the database has a strong ability to maintain and efficient data integration capabilities. On the current market, improve the level of database 
maintenance can take full advantage of market resources, through the purchase of open-source databases and integrated systems to ensure the safety of their own database management. In the daily maintenance management process, you can make good use of the development company data maintenance. In addition, the computer design and development companies need to continue to introduce the compound talents, focusing on the cultivation of professional talents, and absorb the elite to ensure the stability of the company's operations and development. For the entire system to provide adequate management personnel, strengthen the maintenance and management efficiency and reduce the risk of the probability of occurrence.

\section{Conclusion}

The emergence of computers has brought great changes to the modern life and work way, now people have become accustomed to the computer age. Computer has made a great contribution to the community as a whole and the computer not only promotes the economic development, but also promotes the cultural communication. But the achievement of computer performance and function cannot do without the support of computer software. Only good computer software can make the computer function to be played. On the other hand, the database as a computer technology, it plays a significant role in improving the information level of enterprise.

\section{References}

[1] Jia Xinzhang, Li Jingyuan. Computer and Modernization, Vol. 6 (2014) No 53, p.25-26

[2] Peng Sue. China New Technology Products, Vol. 12 (2015) No 27, p.74-76

[3] Qian Xiyuan, Jing Jianfen. Computer Engineering, Vol. 30 (2014) No 19, p.144-145

[4] Wang Li. Computer CD-ROM Software and Application, Vol. 29 (2008) No 27, p.21-23

[5] Zhang Gongxu, Sun Jing. Computer and Modernization, Vol. 8 (2013) No 27, p.57-60 